\title{
Bone Healing Following Different Types of Osteotomy: Scanning Electron Microscopy (SEM) and Three-Dimensional SEM Analyses
}

\author{
Marko Blaskovic, ${ }^{1}$ Dragana Gabrić, ${ }^{1}$ Nichola J. Coleman, ${ }^{2}$ Ian J. Slipper, ${ }^{2}$ Mitko Mladenov, ${ }^{3}$ \\ and Elizabeta Gjorgievska ${ }^{4, *}$ \\ ${ }^{1}$ Department of Oral Surgery, School of Dental Medicine, Faculty of Dentistry, University of Zagreb, Republic of Croatia, Ivana \\ Gundulića 5, 10000, Zagreb, Croatia \\ ${ }^{2}$ Faculty of Engineering and Science, University of Greenwich, Maritime, Gillingham, Chatham ME4 4TB, Central Ave, \\ Gillingham, Chatham ME4, UK \\ ${ }^{3}$ Faculty of Natural Sciences and Mathematics, University "Ss Cyril and Methodius" Skopje, Arhimedova, 1000 Skopje, \\ Republic of Macedonia \\ ${ }^{4}$ Faculty of Dentistry, University "Ss Cyril and Methodius" Skopje, Vodnjanska 17, 1000 Skopje, Republic of Macedonia
}

\begin{abstract}
The aim of the present study was to compare dynamics of the bone healing process after different types of osteotomies. In total, 24 Wistar rats were subjected to different types of osteotomy performed with standard steel bur, piezosurgery, contact, and non-contact Erbium:yttrium-aluminum-garnet (Er:YAG) laser ablation. The animals were randomly divided into four groups, to be euthanized immediately after the procedure, or at 1, 2, or 3 weeks after surgery. The obtained bone samples were analyzed by scanning electron microscopy (SEM). Immediately after surgery, there were significant differences in the appearance of the bone defects, with presence of bone fragments and debris after standard steel bur preparation, compared with the clean smooth walls and relatively sharp edges in all other groups. The initial bone formation in defects prepared by piezosurgery was observed to be the most rapid. After 3 weeks, all bone defects were completely restored; although, differences in the healing pattern were noted, with a modest initial delay in healing after laser preparation. The first stage of the bone healing process was delayed when contact and non-contact Er:YAG laser modes were used and accelerated by piezosurgery; however, the results after 3 weeks demonstrated similar restitution of defects in all tested groups.
\end{abstract}

Key words: animal models, laser, piezoelectric surgery, bone healing

\section{INTRODUCTION}

Osteotomies and ostectomies are frequently performed procedures in oral and maxillofacial surgery. They are generally performed with traditional instruments such as diamond or steel burs, oscillating saws, chisels, or mills (Rupprecht et al., 2003; Stopp et al., 2008). Despite the fact that they are considered a golden standard for bone osteotomies, these instruments present some disadvantages caused by the pressure and friction arising during their contact with the bone tissue. Thin and fragile bone segments can fracture because of the applied contact pressure and vibrations. Excessive friction can also lead to thermal damage and mechanical trauma of the mineralized tissue (Gabrić Pandurić et al., 2012). Furthermore, the deposition of metal shavings, biomechanical stress, potential soft tissue trauma and dispersal of bony particles and debris into surrounding soft tissue and osteotomy walls can delay, or even, jeopardize the healing process (Barone et al., 1997; Kerawala et al., 1999; Kondo et al., 2000; Rupprecht et al., 2003; Gabrić Pandurić et al., 2012).

Consequently, the requirement for superior methods of bone tissue surgery, which would enable less invasive, precise osteotomies unrelated to the geometry of the mechanical

${ }^{*}$ Corresponding author. egjorgievska@stomfak.ukim.edu.mk instrument, with limited collateral tissue trauma and reduced force and friction, has prompted the development of new osteotomy techniques: erbium laser ablation and piezoelectric surgery (Sulewski, 2000; Frentzen et al., 2003; Stübinger et al., 2007; de Mello et al., 2008; Romeo et al., 2009; Stübinger et al., 2009; Esteves et al., 2013).

Piezoelectric bone surgery is a novel ultrasonic technique for osteotomy and osteoplasty, operating within a frequency range of $24-32 \mathrm{kHz}$. It is characterized by selective cutting of the mineralized tissue without affecting the adjacent soft tissue, even in the case of accidental contact (Esteves et al., 2013).

The micrometric vibrations produced during application of the instrument enable a precise cutting action with increased intraoperative control, especially in anatomically difficult areas. In addition, the minimal noise production during bone ablation contributes to the reduction of stress and fear among patients during surgical procedures. Furthermore, less force is necessary by the operator to obtain a cut in the bone compared with that required during the application of a standard rotational bur (Vercellotti, 2004; Vercellotti et al., 2005; Sohn et al., 2007; Gonzales-Garcia et al., 2008).

Erbium:yttrium-aluminum-garnet (Er:YAG) lasers are suitable for bone ablation due to their high absorption rate by water, one of the main components of vital bone. The Er:YAG laser emits a wavelength of $2.94 \mu \mathrm{m}$ which is almost entirely 
absorbed by water and, to a much lower extent, by hydroxyapatite minerals (Convissar, 2004; Iaria, 2008; Kang et al., 2008), allowing efficient bone ablation with little or no carbonization phenomenon (Stübinger, 2010; Gabrić Pandurić et al., 2012; Pandurić et al., 2014). After the laser irradiation onset, the absorbed energy is converted into heat, causing vaporization of interstitial water and internal pressure build up. When the pressure exceeds the hydroxyapatite crystal strength, disruptive expansion of the tissue causes microexplosions which cavitate the bone (Stübinger, 2010; Pandurić et al., 2014). Er:YAG laser surgery has many advantages, such as narrow and precise cutting geometry, reduced risk of adjacent tissue injury, high bactericidal and detoxification effects, absence of metal abrasion, reduced tissue bleeding, and lack of vibration during procedures (Pandurić et al., 2014).

It should be noted that the periphery of the laser beam is of lower energy than its central section, transmitting insufficient energy for effective bone ablation. Instead, the energy from the periphery of the beam causes cumulative heat deposition causing a thermally altered layer of bony tissue to a maximum depth of $\sim 30 \mu \mathrm{m}$ (Choi \& Welch, 2001; Sasaki et al., 2002a, 2002b; de Mello et al., 2008). This thermally changed layer is considered to be harmless with regard to the subsequent bone healing process (Yoshino et al., 2009).

Currently, there is a limited number of studies which compare novel methods for bone osteotomies, such as piezoelectric and laser surgery, with standard bur osteotomy in terms of bone reparation. Therefore, the purpose of the present Wistar rat study was to determine the dynamics of the bone healing process following osteotomies performed by standard steel bur, piezoelectric surgery, and Er:YAG laser ablation in contact and non-contact modes. The null hypothesis tested was that there were no differences between the different surgical techniques with respect to neoformation of bone in the artificially prepared osteotomies.

\section{Material And Methods}

This study was performed in compliance with the Ethical Committee of the University "Ss Cyril and Methodius" in Skopje. This research was approved by the School of Dental Medicine, University of Zagreb and the Animal Ethics Committee of the Faculty of Natural Sciences and Mathematics, University of Skopje. In addition, the experiments undertaken in the study complied with the ARRIVE guidelines and were carried out in accordance with the UK Animals (Scientific Procedures) Act, 1986 and associated guidelines, EU Directive 2010/63/EU for animal experiments, or the National Institutes of Health guide for the care and use of Laboratory animals (NIH Publications No. 8023, revised 1978).

\section{Surgical Procedure}

A total of 24 Wistar rats (Rattus norvegicus albinus) were used in this study. The animals were 10 weeks old with an average weight 300-350 g. One week before the experiment, the animals were allowed to acclimatize in a controlled environment with a room temperature of $22^{\circ} \mathrm{C}, 12 \mathrm{~h}$ light/dark cycle, and access to water and food ad libitum. The animals were randomly divided into four groups, each group consisting of six rats. The groups were based on the postoperative duration of survival of the animals: group 1 was sacrificed on the day of the surgery; group 2 was sacrificed 7 days after the surgery; group 3 was sacrificed 14 days after the surgery; and group 4 was sacrificed 21 days after the surgery.

All animals were subjected to the same surgical procedure after application of general anesthesia by an intraperitoneal injection of thiopental sodium (RhonePoulenc Rorer Limited, Nenagh, Co Tipperary, Ireland). The animals were positioned in dorsal decubitus, before hair-removal and disinfection with povidone iodine solution on the surgical sites on both tibiae. A linear incision, $18 \mathrm{~mm}$ long, was made through the skin, muscles, and periosteum, on each tibia. The bone was exposed by gentle reflection of the dermo-periosteal flap. Four osteotomies were performed always in the same sequence: left proximal by surgical bur, left distal by digitally controlled non-contact Er: YAG laser, right proximal by piezosurgery, and right distal by a contact Er:YAG laser. The osteotomies were $5 \mathrm{~mm}$ away from each other and $\sim 2 \mathrm{~mm}$ deep, with a diameter ranging between 1.0-2.0 $\mathrm{mm}$ depending on the instrument applied. All the osteotomies were performed by the same surgeon.

\section{Surgical Bur Osteotomy}

A low-speed handpiece at $1,200 \mathrm{rpm}$ and a new round steel surgical bur with $2 \mathrm{~mm}$ diameter under copious irrigation was used to perform the proximal osteotomy on the left tibia.

\section{Piezosurgery Osteotomy}

A Piezomed (W\&H Dentalwerk Burmoos GmbH, Burmoos, Austria) device set at maximum power with continuous water cooling using a round diamond tip ( $1.2 \mathrm{~mm}$ diameter) was used to perform the proximal osteotomy on the right tibia. The average pressure applied during the instrument handling was $15 \mathrm{~N}$.

\section{Er:YAG Contact Mode Osteotomy}

An Er:YAG LightWalker ${ }^{\circledR}$ laser system (Fotona, Ljubljana, Slovenia) with a H-14N handpiece and fiber of $1.0 \mathrm{~mm}$ diameter (core diameter of $940 \mu \mathrm{m}$ ), with continuous water spray $(40-60 \mathrm{~mL} / \mathrm{min})$ was used to perform the distal osteotomy on the right tibia. The following parameters were used: power $7.5 \mathrm{~W}$, pulse energy $375 \mathrm{~mJ}$, repetition rate $20 \mathrm{~Hz}$, medium-short pulse mode (pulse duration $10 \mu \mathrm{s}$ ).

\section{Er:YAG Non-Contact Mode Osteotomy}

An Er:YAG LightWalker ${ }^{\circledR}$ laser system (Fotona) with a recently developed digitally controlled $2 \mathrm{~mm}$ diameter circular handpiece (X-Runner ${ }^{\text {TM }}$, Fotona, Slovenia) with water 
spray set at ratio $4: 4$ and focal distance of $13 \mathrm{~mm}$ was used to perform the distal osteotomy on the left tibia. The following parameters were used: power $7.5 \mathrm{~W}$, pulse energy $750 \mathrm{~mJ}$, repetition rate $10 \mathrm{~Hz}$, quantum-square pulse mode (QSP, a train of five short pulses of $50 \mu$ s separated by $85 \mu \mathrm{s})$.

Biosecurity standards to protect the personnel were used during both groups of laser osteotomies.

At the end of the surgery, all incisions were closed with interrupted absorbable sutures (Vicryl, 6.0; Ethicon Inc., Cincinnati, $\mathrm{OH}, \mathrm{USA}$ ) except for the animals from the first group. They were euthanized immediately after performing all the osteotomies within this group, with an overdose of the previously administered anesthetic solution.

All other animals were treated with $1 \mathrm{mg} / \mathrm{kg}$ veterinary acetaminophen in $1 \mathrm{~L}$ of water. The animals from the second group were sacrificed after 1 week, from the third group after 2 weeks, and from the fourth group after 3 weeks, again with an anesthetic overdose.

\section{Scanning Electron Microscopy (SEM)}

The remnants of soft tissue were removed from the bone samples collected from the rat tibiae which were then cut transversely to contain the complete osteotomy sites. Each uncoated sample was mounted onto an aluminum stub and pumped down in a vacuum desiccator to remove excess moisture prior to SEM analysis. Secondary electron images were obtained with a cold cathode field-emission gun scanning electron microscope (FEG-SEM Hitachi SU 8030, Tokyo, Japan).
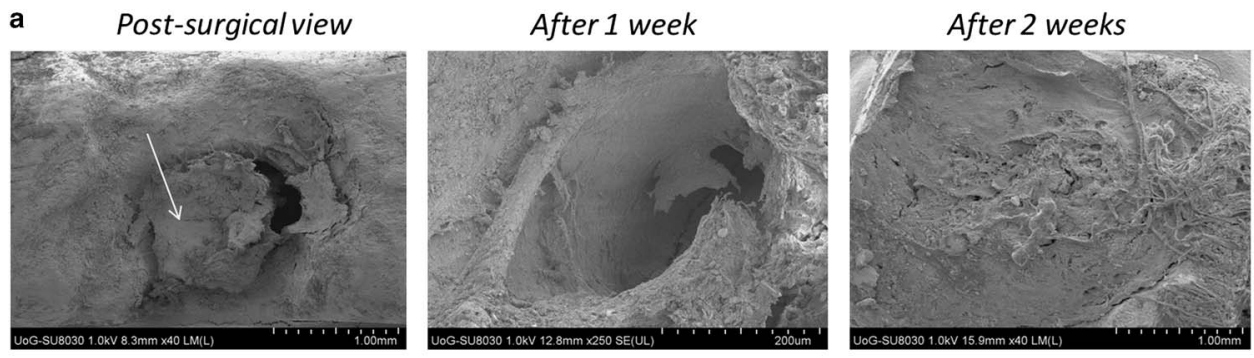

b
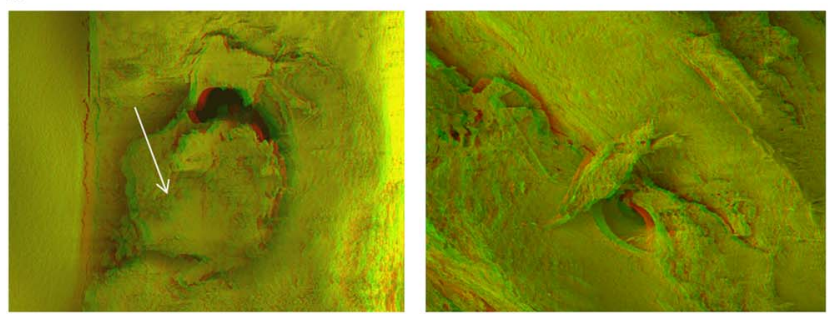

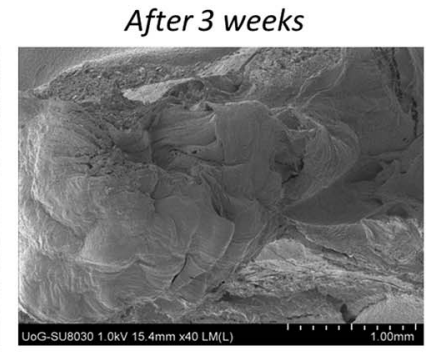

\section{SEM/Three-Dimensional Stereo- Photographs (ANAGlyphS) (3D-SEM)}

The 3D stereo-photographs (anaglyphs) were created by taking two stereo-pair photographs with a separation of $7^{\circ}$, the first at $-3.5^{\circ}$ and the second with $a+3.5^{\circ}$ tilt. A red filter was used for the lower angle (left eye), and a green filter was used for the higher angle (right eye). The microphotographs were superimposed and visualized with 3D-anaglyphic glasses, as described previously by Gjorgievska et al. (2013).

\section{RESULTS}

The SEM/3D micrographs of the osteotomies prepared with a standard steel bur (Fig. 1) immediately after surgery showed the presence of a blood clot, which was retracted due to partial desiccation of the sample during preparation for SEM analysis. The presence of debris on the cavity walls was also evident. After 1 week there were initial signs of new bone formation at the bottom of the bone defect, whereas after 2 weeks the defect was completely repaired. Moreover, after 3 weeks the presence of abundant new bone could be observed extending beyond the perimeter of the original defect site.

Figure 2 demonstrates that after this type of osteotomy, immediately after surgery, red blood cells as well as debris were attached to the cavity walls; whereas up to 3 weeks later the bone repair was characterized by the presence of multiple collagen fibers and osteoblasts in the defect site.

The micrographs of osteotomies performed by piezosurgery (Fig. 3) displayed a bone defect with a retracted blood clot penetrating into the bone marrow immediately after the surgical procedure. The irregular, although sharp, edges of
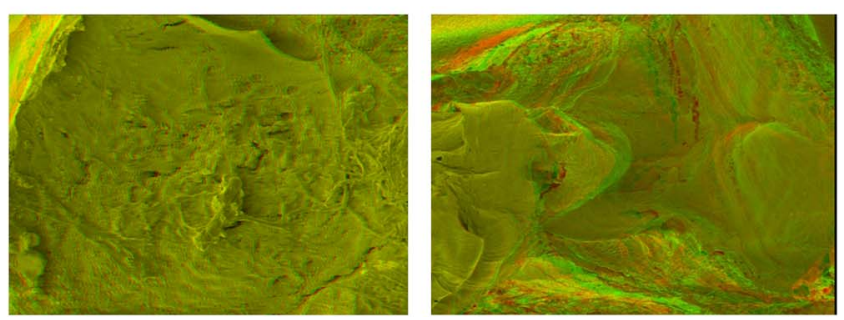

Figure 1. a: Scanning electron microscopy (SEM) and (b) Three-dimensional SEM (anaglyphs) of osteotomies prepared by standard steel bur in respect of time (immediately after the surgery up to 3 weeks after the surgery): postsurgical view-presence of a blood clot (which is retracted due to desiccation of the samples during preparation for the SEM) and debris on the cavity walls; after 1 week-beginning of a new bone formation at the bottom of the bone defect; after 2 weeks-the defect is completely restituted; after 3 weeks-presence of abundant new bone formation which grows out of the defect. 

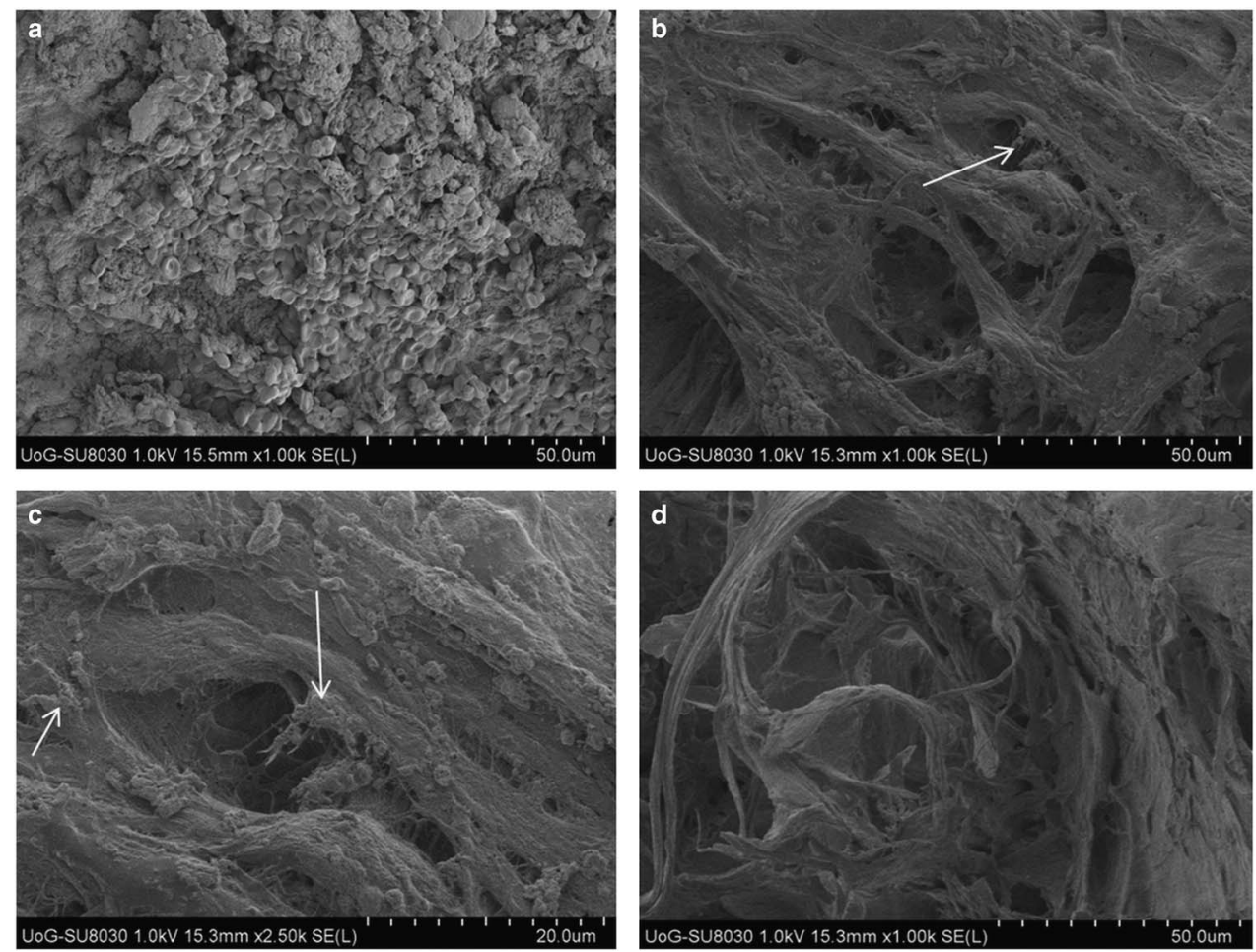

Figure 2. Bone formation after osteotomy performed by a standard steel bur in respect of time: (a) Immediately after surgery-red blood cells, as well as debris attached to the cavity walls, are present; (b-d) Up to 3 weeks-presence of multiple collagen fibers and osteoblasts (arrows) in the defect.
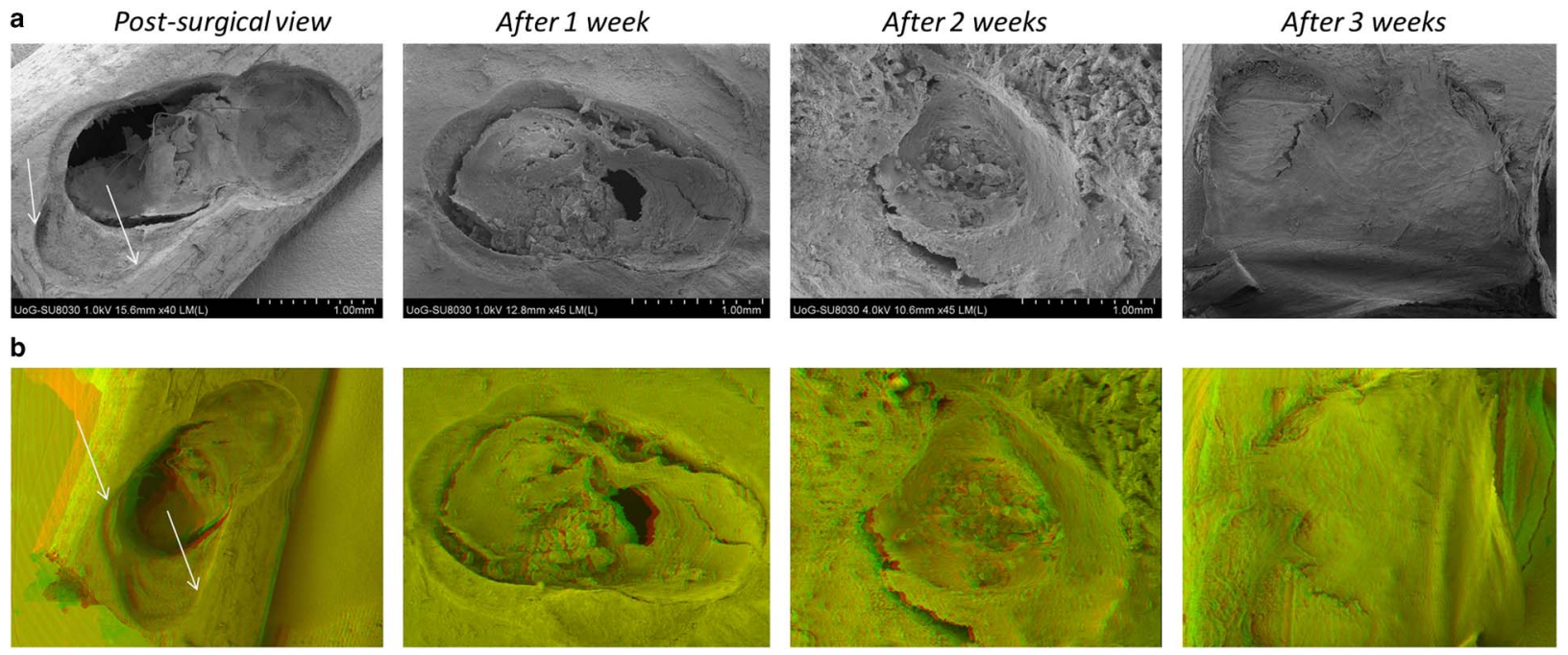

Figure 3. a: Scanning electron microscopy (SEM) and (b) Three-dimensional SEM (anaglyphs) of osteotomies prepared by piezosurgery in respect of time (immediately after the surgery up to 3 weeks after the surgery): postsurgical view - a bone defect with a retracted blood clot penetrating into the bone marrow, irregular edges of the defect due to oscillations of the piezoelectric device during the preparation; after 1 and 2 weeks-bone formation; after 3 weeksthe defect is completely repaired.

the defect, were due to oscillations of the piezoelectric device during the preparation.

Figure 4 shows that after 1 week, initial bone formation was observed; and in subsequent micrographs up to 3 weeks, collagen fibers and osteoblast proliferation were visible, which indicate new bone formation in the defect.
The osteotomies prepared with contact mode Er:YAG laser (Fig. 5) displayed a bone defect with a retracted blood clot (due to the laser's coagulation effect) which penetrated into the bone marrow. Immediately after surgery, clean cavity walls, devoid of debris, were visible. The edges of the defect were sharp and well defined. After 1 and 2 weeks, 

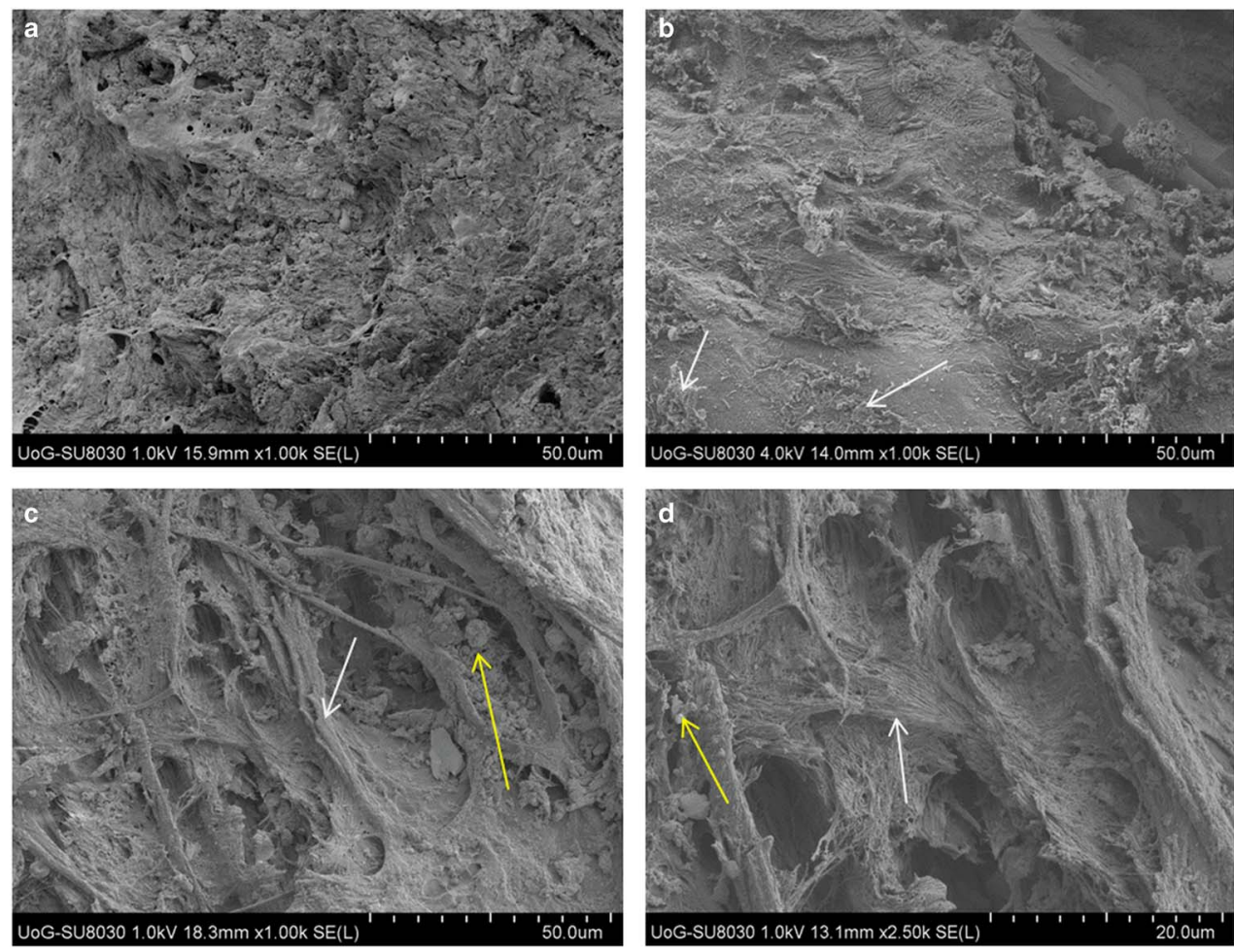

Figure 4. Bone formation after osteotomy performed by piezosurgery in respect of time: (a) Immediately after surgery-irregular surface present at the cavity walls, probably as a result of the microexplosions during the preparation; (b) After 1 week-initial bone formation, osteoblast adhesion (white arrows); (c,d) Up to 3 weeks-collagen fibers and osteoblast proliferation in the defect, mineral incorporation into the collagen mesh (yellow arrows).
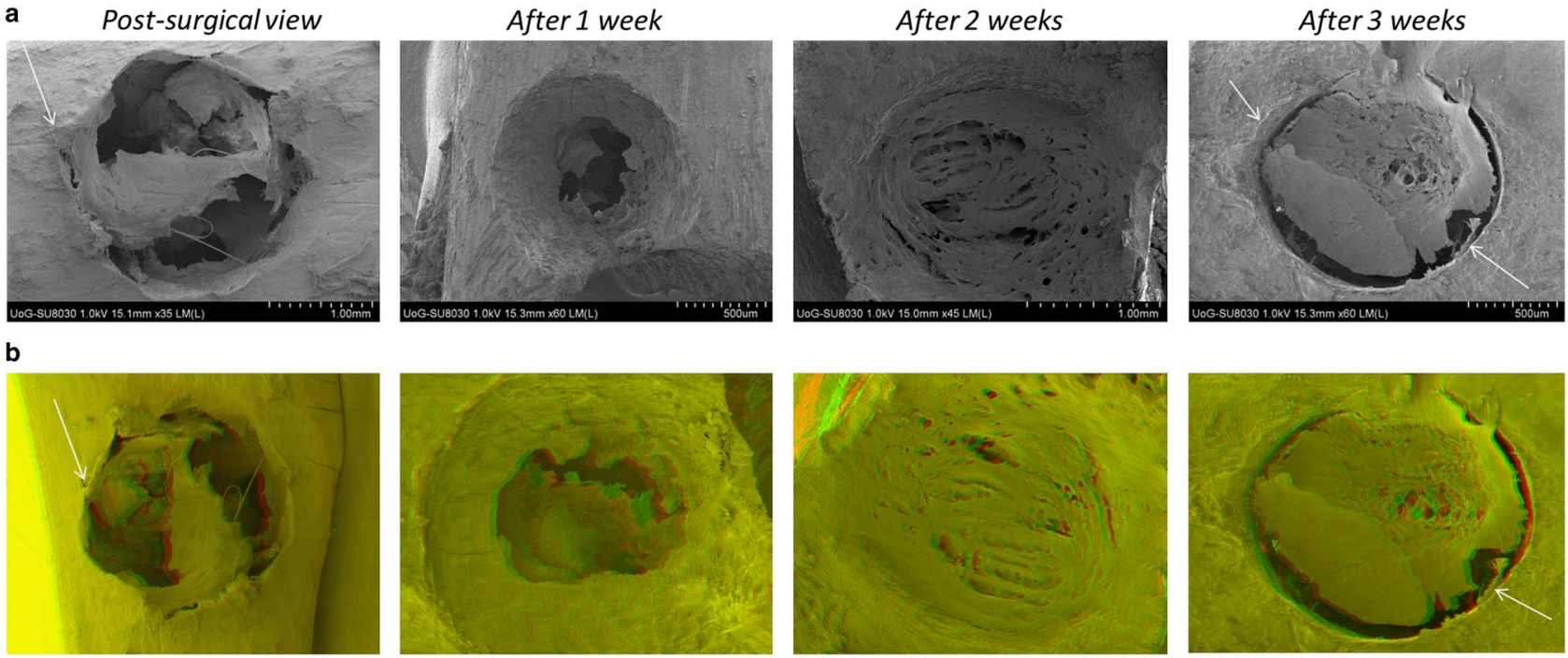

Figure 5. a: Scanning electron microscopy (SEM) and (b) Three-dimensional SEM (anaglyphs) of osteotomies prepared with contact mode Erbium:yttrium-aluminum-garnet laser in respect of time (immediately after the surgery up to 3 weeks after the surgery): immediately after surgery-a bone defect with a retracted blood clot (due to the laser's coagulation effect) penetrating into the bone marrow, the edges of the defect are sharp and well defined; after 1 and 2 weeks-progressive bone formation; after 3 weeks - complete restitution of the defect, although a visible line is present, probably as a result of the thermal effect of the laser irradiation on the bone tissue (carbonization).

progressive bone formation had occurred; whereas after 3 weeks, complete restitution of the defect was apparent. In addition, a visible "rim" was present around the perimeter of the defect, which is likely to have arisen from the thermal "carbonization" effect of the laser irradiation on the bone tissue. Also, the hemostatic effect of the laser leads to 
occurrence of the previously described retracted blood clot, with only a small number of erythrocytes present above this carbonized layer, which may account for the slower bone recovery in the first 2 weeks.

In Figure 6, after 1 week, initial bone formation was observed to have commenced. Within 2 weeks, the beginnings of a fibrin mesh were visible, although the laser-ablated surface was still evident in some areas. By 3 weeks, the collagen fibers and osteoblasts had proliferated into the defect, and minerals had been incorporated in the organic matrix of the newly formed bone.

The non-contact mode Er:YAG laser (Fig. 7) created a bone defect with a remnant of spongious bone at the base of the cavity, penetrating into the bone marrow. The edges of the defect were sharp and well defined, although the bottom of the preparation was wider in comparison with that of the contact Er:YAG laser osteotomy. The micrographs showed delayed restitution, where after 1 week absence of bone formation was evident. By 2 weeks, bone neoformation was observed at the base of the cavity. After 3 weeks, these samples showed significant restitution of the defect, with a preserved rim of carbonization, typical of cortical bone. The carbonized rim observed after non-contact laser osteotomy was more pronounced than that produced in contact mode. The bone formation following non-contact laser osteotomy was delayed as a result of the carbonization, although erythrocytes were observed to readily populate the carbonized area of bone. As time progressed, the new bone proliferation advanced and collagen fibers were visible. After 3 weeks following surgery, a solid bone was formed (Fig. 8).

\section{Discussion}

The present in vivo study performed on animal models concerns the progression and dynamics of the bone healing process following three novel preparation methods for bone osteotomies: piezosurgery and Er:YAG laser ablation in contact and non-contact modes, in comparison with the standard steel bur osteotomy.

The basic mechanism of bone repair after osteotomy involves the presence of a hematoma contained by the surrounding tissues, after which a fibrinous blood clot is formed to serve as a medium for repair and cell ingrowth (Shapiro, 2008). The first few days postsurgery are characterized by an inflammatory phase. Bone repair begins by laying down intramembranous woven bone, whereas the next stage of the repair process involves transformation of the newly woven bone into lamellar bone.

During the current study, the presence of a blood clot was observed alongside the debris of the smear layer on the cavity walls in the osteotomies produced with the standard steel bur.
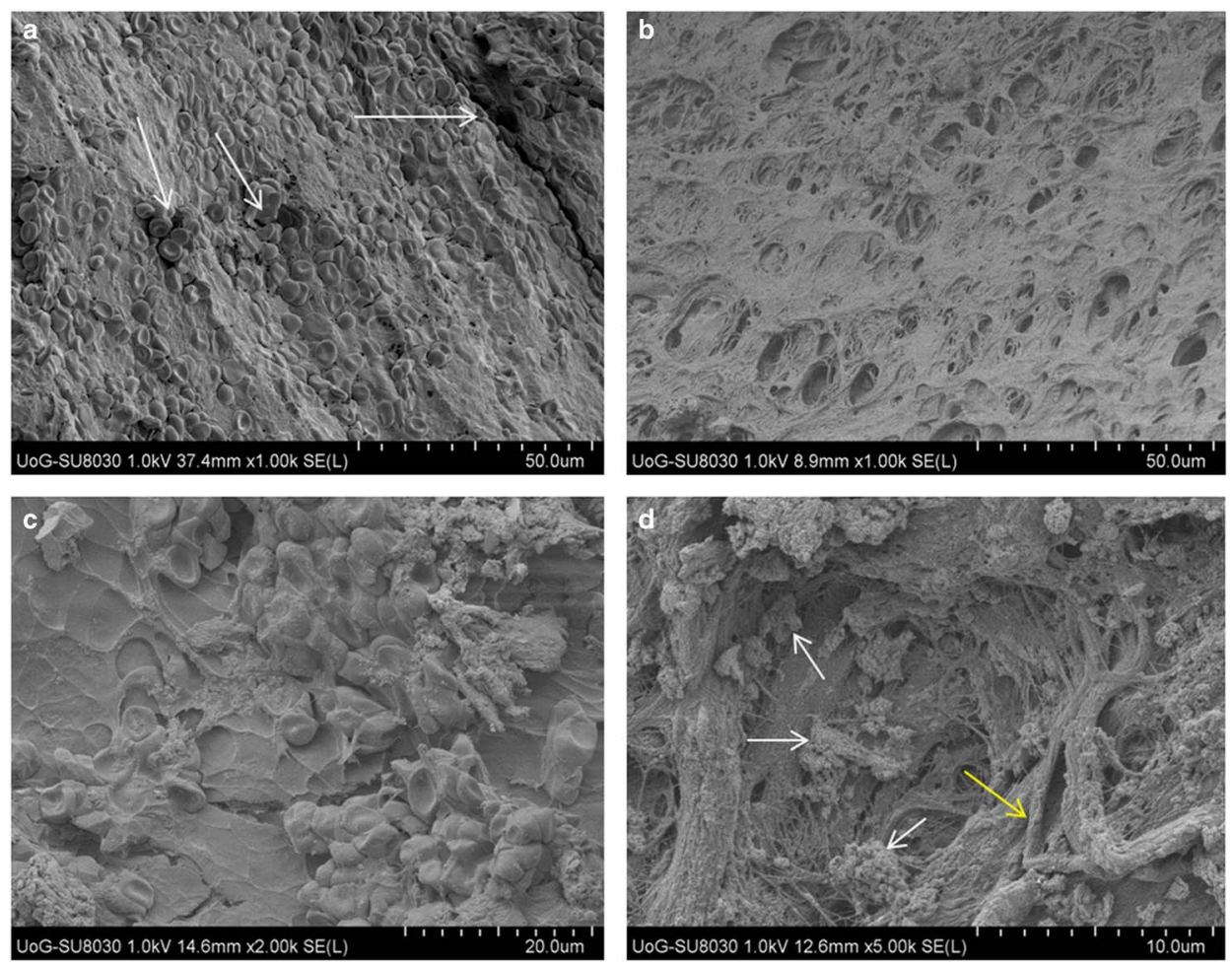

Figure 6. Bone formation after osteotomy performed by contact mode Erbium:yttrium-aluminum-garnet laser in respect of time: (a) Immediately after surgery_clean cavity walls, without any debris, erythrocytes present; areas of carbonization (arrows); (b) After 1 week—initial bone formation, fibrin mesh evident; (c) After 2 weeks-bone proliferation, osteoblasts, and erythrocytes visible, penetration of collagen fibers through the carbonization layer in order to form the collagen network; (d) after 3 weeks-collagen fibers (yellow arrows) and osteoblast proliferation in the defect, incorporation of minerals in the organic matrix (white arrows). 

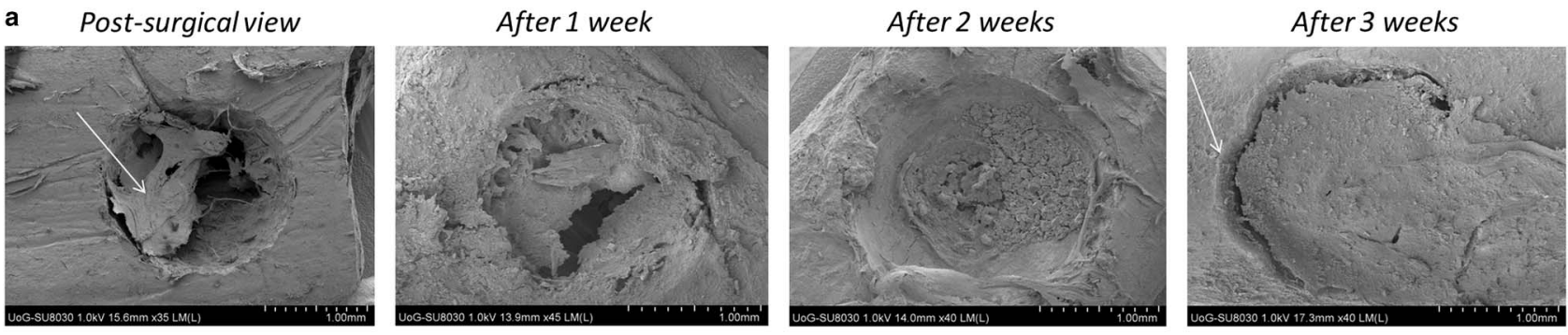

b
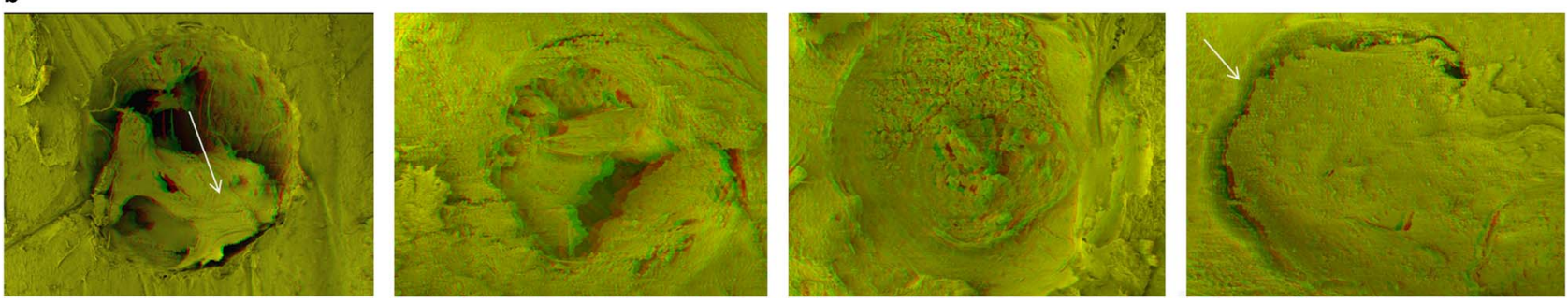

Figure 7. a: Scanning electron microscopy (SEM) and (b) Three-dimensional SEM (anaglyphs) of osteotomies prepared with non-contact mode Erbium:yttrium-aluminum-garnet (Er:YAG) laser (x-runner) in respect of time (immediately after the surgery up to 3 weeks after the surgery): postsurgical view-a bone defect with presence of retracted blood clot, the edges of the defect are sharp and well defined, although less regular than the contact mode Er:YAG laser, the preparation cavity has equal diameter on the surface and in the bottom; after 1 week-absence of bone formation; after 2 weeks - woven bone formation at the bottom of the cavity; after 3 weeks-almost complete restitution of the defect.
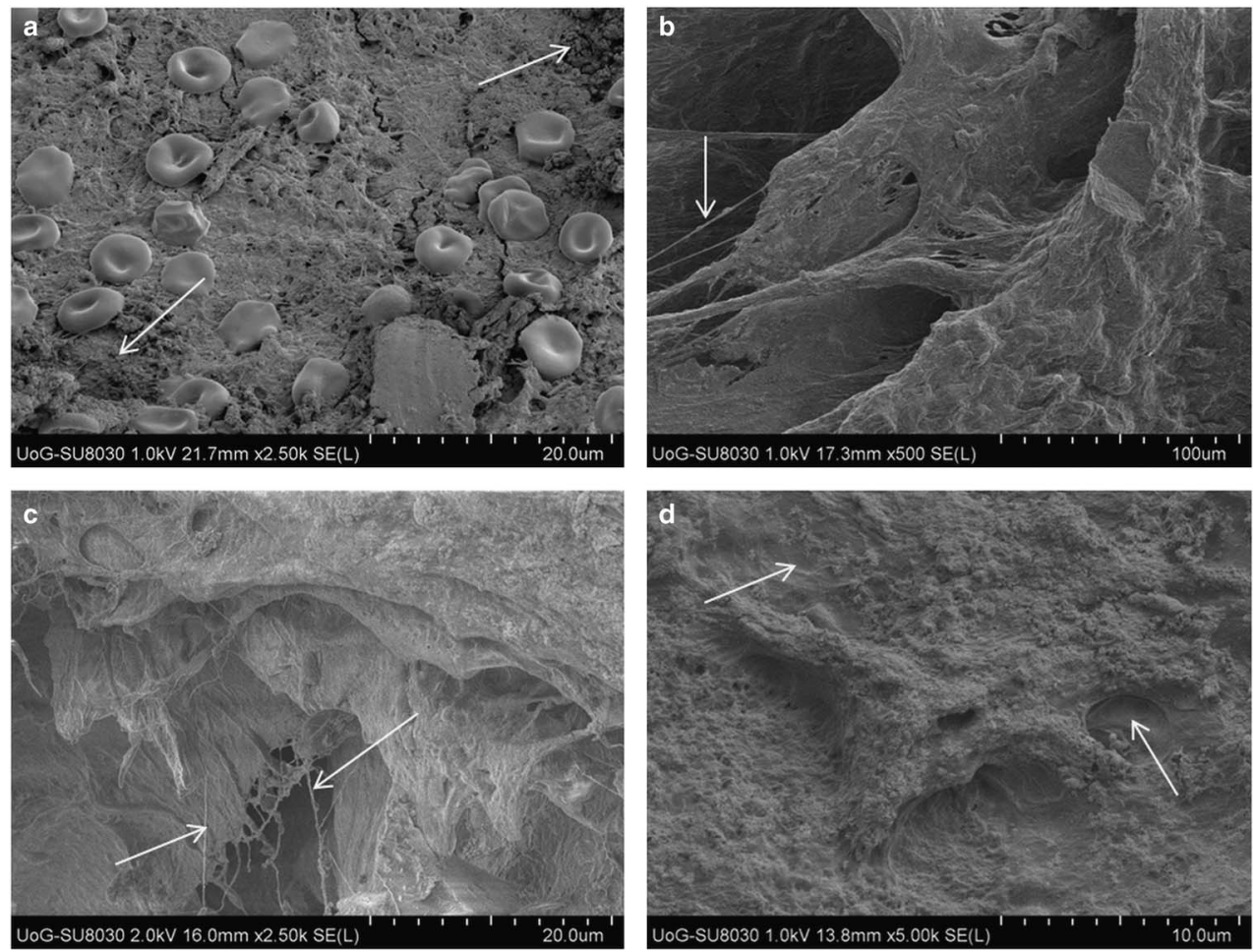

Figure 8. Bone formation after osteotomy performed by non-contact mode Erbium:yttrium-aluminum-garnet laser (x-runner) in respect of time: (a) Immediately after surgery- rare erythrocytes above the carbonization layer (arrows toward the carbonized tissue); (b,c) After 1 and 2 weeks-bone proliferation, collagen fibers are visible (arrows); (d) After 3 weeks - solid bone is formed, although areas of carbonization are still present (arrows).

In this case, the presence of debris did not appear to have a detrimental impact on the bone healing process. Gabrić Pandurić et al. (2012) and Romeo et al. (2009) also reported that bone fragments and fiber-like debris were found on the bone surface after the use of rotating instruments, which they reported to be a potential cause of delayed healing. 
Another study, contradictory to the previous ones, stated that the bone debris provided an important contribution to osseointegration, mediating the connection between the bone and implant surface (Mainetti et al., 2015).

Unlike traditional manual bone cutting instruments, piezoelectric bone surgery offers many advantages, which include: precise cutting and superior safety; lower force of application and greater control of the device; reduced necrosis; selective tissue cutting and minimal operative invasion, as the hard and soft tissues are cut at different frequencies. This technique also benefits from a bleed-free surgery site, as a cavitation effect creates bubbles in the physiological fluid which implode to generate shockwaves which cause microcoagulation. Enhanced bone regeneration and superior healing processes are also associated with piezoelectric surgery, as oxygen released during cutting has an antiseptic effect and the ultrasound vibration is reported to stimulate bone cell metabolism (Rahnama et al., 2013).

The most significant disadvantages of piezoelectric surgery are the increased operation time that is required for bone preparation and the precise control of irrigation, in order to regulate the effect of cavitation, and also to avoid overheating (Rahnama et al., 2013). The present study found that the osteotomy defect after piezosurgery has irregular, smooth walls. Unlike the group treated with a standard steel bur, in the group whose osteotomy was performed by piezosurgery, neither signs of bone fragments nor traces of debris were found in the defects immediately after surgery. This finding is in agreement with those of previous studies, which have shown that piezosurgery produces clean and precise osteotomies with smooth walls and decreased bleeding (Esteves et al., 2013). In a recent study, Esteves et al. (2013) report that there were no observed differences in the bone healing process when piezoelectric surgery was compared with conventional drill osteotomy in rat tibiae. Conversely, according to Preti et al. (2007) more active osteogenesis was reported when sites were prepared with piezoelectric surgery rather than traditional osteotomy. The findings of the current study confirm this observation, as it has been demonstrated that a more pronounced initial progression of bone formation following piezoelectric osteotomy appears relative to that of the standard steel bur.

Er:YAG laser ablation is precise and produces well-defined sharp edges and regular borders with the surrounding tissue. Wound healing is observed to be comparable with or even faster than that of osteotomies performed with drills or oscillating saws (Pandurić et al., 2014). The well-defined edges of the preparation and the unique surface which is absent of a smear layer is considered to enhance the adhesion of blood elements at the start of the healing process (Sasaki et al., 2002a; Sasaki et al., 2002b; Stübinger, 2010). It is already acknowledged that the smear layer may act as a barrier which obstructs blood element interaction with the underlying tissue, resulting in a prolonged healing process (Pandurić et al., 2014).

Conversely, contact Er:YAG laser surgery is associated with a strong hemostatic effect, which consequently leads to reduced formation of the blood clot. Therefore, a modest reduction in the first stages of the bone healing process can be anticipated after this procedure. This reduced initial healing was evident after 1 week postsurgery in this study; which was then followed by an improvement in the quantity of subsequent bone neoformation.

The use of the Er:YAG laser results in an $\sim 30 \mu$ m-thick altered layer on the margins of the osteotomy. This layer is composed of a superficial part without clear structures and an underlying layer with minimal thermal damage. Histologically, the changed (carbonization) layer consists of a lightly stained superficial layer without defined structures and a deep, less affected layer (Gabrić Pandurić et al., 2012). An important fact is that this carbonization zone persists in the later stages of the bone repair process for up to 105 days (el Montaser et al., 1997). Our results indicate that the presence of this carbonization layer did not entirely inhibit bone neoformation. In fact, the collagen fibers penetrated through the carbonization layer in order to produce the collagen network as a basis for the new bone formation.

The present study showed a modest initial delay in bone healing when contact and non-contact Er:YAG laser modes were used to perform the osteotomies, principally due to the hemostatic effect. Despite differences in the dynamics of bone healing, after 3 weeks the extent of bone formation did not differ significantly among the different surgical methods.

\section{CONCLUSIONS}

This study compares the rates and extents of bone healing in osteotomies performed on rat tibiae with a standard steel bur, piezoelectric surgery and laser Er:YAG ablation in both contact and non-contact modes. Piezoelectric surgery is a precise ultrasonic technique which can be used to selectively cut either soft tissues (at high frequencies) or hard tissues (at low frequencies). Er:YAG laser ablation cuts by evaporating the water in the bone tissue which causes destructive microexplosions. Both piezoelectric surgery and Er:YAG laser ablation are associated with hemostatic effects, minimal bleeding, and reduced patient discomfort.

Osteotomies produced with a standard steel bur exhibited a roughened surface with a smear layer of debris on the cavity walls. Conversely, the defects prepared by piezosurgery and laser ablation were characterized by smooth cavity walls and the absence of detritus. The initial healing process of the defects prepared by piezosurgery was observed to be the most rapid. Osteotomies prepared by contact and non-contact Er:YAG laser modes exhibited an initial delay in the bone healing process, arising from the pronounced hemostatic effect; however, the results after 3 weeks demonstrated similar restitution of the defects in all of the tested groups. This study supports the view that piezosurgery and Er:YAG laser ablation can be a potential benefit in performing dental osteotomies. 


\section{REFERENCES}

Barone, C.M., Jimenez, D.F., Yule, G.J. \& Strauch, B. (1997). Analysis of bone formation after cranial osteotomies with a high-speed drill. J Craniofac Surg 8, 466-470.

Chol, B. \& Welch, A.J. (2001). Analysis of thermal relaxation during laser irradiation of tissue. Lasers Surg Med 29, 351-359.

CONVISSAR, R.A. (2004). The biologic rationale for the use of lasers in dentistry. Dent Clin North Am 48, 771-794.

de Mello, E.D., Pagnoncelli, R.M., Munin, E., Filho, M.S., de Mello, G.P., Arisawa, E.A. \& De Oliveira, M.G. (2008). Comparative histological analysis of bone healing of standardized bone defects with the Er:YAG laser and steel burs. Lasers Med Sci 23, 253-260.

el Montaser, M.A., Devlin, H., Sloan, P. \& Dickinson, M.R. (1997). Pattern of healing of calvarial bone in the rat following application of the erbium-YAG laser. Lasers Surg Med 21, 255-261.

Esteves, J.C., Marcantonio, E. Jr, De Souza Faloni, A.P., Rocha, F.R., Marcantonio, R.A., Wilk, K. \& Intini, G. (2013). Dynamics of bone healing after osteotomy with piezosurgery or conventional drilling-histomorphometrical, immunohistochemical, and molecular analysis. J Transl Med 11, 221.

Frentzen, M., Götz, W., Ivanenko, M., Afilal, S., Werner, M. \& Hering, P. (2003). Osteotomy with 80-micros $\mathrm{CO}_{2}$ laser pulseshistological results. Lasers Med Sci 18, 119-124.

Gabrić Pandurić, D., Bago, I., Katanec, D., Zabkar, J., Miletić, I. \& ANIĆ, I. (2012). Comparison of Er:YAG laser and surgical drill for osteotomy in oral surgery: An experimental study. J Oral Maxillofac Surg 70, 2515-2521.

Gjorgievska, E.S., Nicholson, J.W., Apostolska, S.M., Coleman, N.J., Booth, S.E., Slipper, I.J. \& Mladenov, M.I. (2013). Interfacial properties of three different bioactive dentine substitutes. Microsc Microanal 19, 1450-1457.

Gonzales-Garcia, A., Diniz-Freitas, M., Somoza-Martin, M. \& Garcia-Garcia, A. (2008). Piezoelectric and conventional osteotomy in alveolar distraction in a series of 17 patients. Int $J$ Oral Maxillofac Implants 23, 891-896.

IARIA, G. (2008). Clinical, morphological, and ultrastructural aspects with the use of Er:YAG and Er,Cr:YSGG lasers in restorative dentistry. Gen Dent 56, 636-639.

KanG, H.W., ОH, J. \& WelCh, A.J. (2008). Investigations on laser hard tissue ablation under various environments. Phys Med Biol 53, 3381-3390.

Kerawala, C.J., Martin, I.C., Allan, W. \& Williams, E.D. (1999). The effects of operator technique and bur design on temperature during osseous preparation for osteosynthesis self-tapping screws. Oral Surg Oral Med Oral Pathol Oral Radiol Endod 88, 145-150.

Kondo, S., OKada, Y., IseKi, H., Hori, T., TaKaKURA, K., Kobayashi, A. \& Nagata, H. (2000). Thermological study of drilling bone tissue with a high-speed drill. Neurosurg 46, 1162-1168.

Mainetti, T., Lang, N.P., Bengazi, F., Sbricoli, L., Soto Cantero, L. \& BotTicelli, D. (2015). Immediate loading of implants installed in a healed alveolar bony ridge or immediately after tooth extraction: An experimental study in dogs. Clin Oral Implants Res 26, 435-441.

Pandurić, D.G., Juric, I.B., Music, S., Molčanov, K., Sušic, M. \& ANIC, I. (2014). Morphological and ultrastructural comparative analysis of bone tissue after Er:YAG laser and surgical drill osteotomy. Photomed Laser Surg 32, 401-408.
Preti, G., Martinasso, G., Peirone, B., Navone, R., Manzella, C., Muzio, G., Russo, C., Canuto, R.A. \& Schierano, G. (2007). Cytokines and growth factors involved in the osseointegration of oral titanium implants positioned using piezoelectric bone surgery versus a drill technique: A pilot study in minipigs. J Periodontol 78, 716-722.

Rahnama, M., Czupkaleo, Ł., Czajkowski, L., Grasza, J. \& Wallner, J. (2013). The use of piezosurgery as an alternative method of minimally invasive surgery in the authors' experience. Videosurg Other Miniinv Tech 8, 321-326.

Romeo, U., Del Vecchio, A., Palaia, G., Tenore, G., Visca, P. \& MagGiore, C. (2009). Bone damage induced by different cutting instruments-an in vitro study. Braz Dent J 20, 162-168.

Rupprecht, S., Tangermann, K., Kessler, P., Neukam, F.W. \& WiLTFANG, J. (2003). Er:YAG laser osteotomy directed by sensor controlled systems. J Craniomaxillofac Surg 31, 337-342.

SASAKI, K.M., AOKI, A., ICHINOSE, S. \& IshiKaWA, I. (2002a). Ultrastructural analysis of bone tissue irradiated by Er:YAG. Lasers Surg Med 31, 322-332.

SAsaki, K.M., Aoki, A., Ichinose, S., Yoshino, T., Yamada, S. \& IsHIKAWA, I. (2002b). Scanning electron microscopy and Fourier transformed infrared spectroscopy analysis of bone removal using Er:YAG and $\mathrm{CO}_{2}$ lasers. J Periodontol 73, 643-652.

SHAPIRO, F. (2008). Bone development and its relation to fracture repair. The role of mesenchymal osteoblasts and surface osteoblasts. Eur Cell Mater 15, 53-76.

Sohn, D.S., Ahn, M.R., Lee, W.H., Yeo, D.S. \& Lim, S.Y. (2007). Piezoelectric osteotomy for intraoral harvesting of bone blocks. Int J Periodontics Restorative Dent 27, 127-131.

Stopp, S., Deppe, H. \& Lueth, T. (2008). A new concept for navigated laser surgery. Lasers Med Sci 23, 261-266.

STÜBINGER, S. (2010). Advances in bone surgery: The Er:YAG laser in oral surgery and implant dentistry. Clin Cosmet Investig Dent $\mathbf{2}$, $47-62$.

Stübinger, S., GhanaAti, S., SAldamli, B., Kirkpatrick, C.J. \& Sader, R. (2009). Er: YAG laser osteotomy: Preliminary clinical and histological results of a new technique for contact-free bone surgery. Eur Surg Res 42, 150-156.

STÜBInger, S., LANDES, C., SeITZ, O. \& SADER, R. (2007). Er:YAG laser osteotomomy for intraoral bone grafting procedures: A case series with a fiber-optic delivery system. J Periodontol 78, 2389-2394.

SulEwski, J.G. (2000). Historical survey of laser dentistry. Dent Clin North Am 44, 717-752.

Vercellotti, T. (2004). Technological characteristics and clinical indications of piezoelectric bone surgery. Minerva Stomatol 53, 207.

Vercellotti, T., Nevins, M.L., Kim, D.M., Nevins, M., WadA, K., SCHENK, R.K. \& FioRellini, J.P. (2005). Osseous response following resective therapy with piezosurgery. Int $J$ Periodontics Restorative Dent 25, 543-549.

Yoshino, T., Aoki, A., Oda, S., Takasaki, A.A., Mizutani, K., Sasaki, K.M., Kinoshita, A., Watanabe, H., Ishikawa, I. \& Izumi, Y. (2009). Long-term histologic analysis of bone tissue alteration and healing following Er:YAG laser irradiation compared to electrosurgery. J Periodontol 80, 82-92. 